\title{
High-performance Flexible Microelectrode Array with PEDOT:PSS Coated 3D Micro-cones for Electromyographic Recording
}

\author{
Jiaao $\mathrm{Lu}^{1}$, Muneeb $\mathrm{Zia}^{1}$, Member, IEEE, Matthew J. Williams ${ }^{2}$, Amanda L. Jacob ${ }^{2}$, Bryce Chung ${ }^{2}$, \\ Samuel J. Sober ${ }^{2}$ and Muhannad S. Bakir ${ }^{1}$, Senior Member, IEEE
}

\begin{abstract}
High signal-to-noise ratio (SNR) electromyography (EMG) recordings are essential for identifying and analyzing single motor unit activity. While high-density electrodes allow for greater spatial resolution, the smaller electrode area translates to a higher impedance and lower SNR. In this study, we developed an implantable and flexible 3D microelectrode array (MEA) with low impedance that enables high-quality EMG recording. With polyimide micro-cones realized by standard photolithography process and PEDOT:PSS coating, this design can increase effective surface area by up to $\mathbf{2 5 0} \%$ and significantly improve electrical performance for electrode sites with various geometric surface areas, where the electrode impedance is at most improved by $\mathbf{9 9 . 3 \%}$. Acute EMG activity from mice was recorded by implanting the electrodes in vivo, and we were able to detect multiple individual motor units simultaneously and with high resolution (SNR $\gg 100)$. The charge storage capacity was measured to be $34.2 \mathrm{mC} / \mathrm{cm}^{2}$, indicating suitability of the electrodes for stimulation applications as well.
\end{abstract}

\section{INTRODUCTION}

Compared with central nervous system recording, electromyographic recordings (EMGs) provide more precise and subtle information about muscle movements, that could be critical for specific motor functions characterization and behavioral disease progression [1]. Traditionally, EMGs are collected by inserting fine wires directly into the muscles [2]. However, fine-wire electrodes predominantly capture bulk EMG consisting of activity of multiple units, whereas single motor units are essential for holistic computational analyses [3], [4]. To acquire single motor unit EMG, two key enablers are low electrode impedance and high spatial resolution. Both are important for analysis methods in neuroscience because low impedance ensures that the signal-to-noise ratio is high so that neural events can be detected and high spatial resolution provides information across different recording sites that is needed to reliably identify events that are generated by the same source. However, these metrics are inherently antagonistic: high spatial resolution demands smaller, densely packed electrode sites whereas electrode impedance is negatively impacted by reduced electrode surface area [5].

One way to address this challenge is to increase the effective surface area (ESA) while maintaining the geometric surface area (footprint). Previous techniques attempting to achieve high ESA for better electrochemical performance can

\footnotetext{
1 Jiaao Lu, Muneeb Zia and Muhannad S. Bakir are with the Department of Electrical and Computer Engineering, Georgia Institute of Technology, Atlanta, GA 30332, USA. jiaaolu@gatech.edu

${ }^{2}$ Matthew J. Williams, Amanda L. Jacob, Bryce Chung and Samuel J. Sober are with the Department of Biology, Emory University, Atlanta, GA 30322, USA.
}

be roughly categorized in two ways: surface modification and $3 \mathrm{D}$ structure fabrication. As for surface modification, coating electrode sites with porous conductive polymer [6], [7], or plasma treatment of the metal surface [8] are some of the ways that have been used to increase the ESA and electrical performance of the electrodes. Fabricating 3D electrodes is another way to increase ESA, however, previously reported methods are either difficult to scale to higher densities or they require relatively complex fabrication processes that are challenging to be adapted on flexible substrate [9]-[13] . Combining surface modification and scalable 3D structure fabrication, this paper aims to achieve high-density microelectrode array (MEA) that is flexible and bio-compatible for in vivo application.

In this work, the 3D electrode sites are fabricated using polyimide micro-cones that are subsequently coated with poly (3,4-ethylenedioxythiophene) polystyrene sulfonate (PEDOT:PSS) to achieve low impedance. We demonstrate the improvement of electrical performance by comparing the impedance between 2D and 3D electrodes with different finishing materials, and show that with the proposed design, the impedance is improved by $99.3 \%$ when compared to the planar gold electrode. In vivo data of the recorded EMG from mouse digastric muscle is also presented; from the measured EMG a high signal-to-noise ratio (SNR) of 447.3 was calculated.

\section{FABRICATION OF 3D MEAs}

The fabrication process of 3D microelectrode arrays (MEAs) is summarized in Fig. 1. At first, a 15- $\mu$ m thick polyimide (PI-2611) layer was spun and cured on a 4-in silicon carrier wafer (Fig. 1(a)). To build 3D polyimide micro-cones, a 15 - $\mu \mathrm{m}$ layer of photodefinable polyimide (HD-8820) was spun, soft baked and patterned by a standard photolithography process in a maskless aligner (Fig. 1(b)). The patterned 3D micro-cones were subsequently cured in oven. A reactive ion etch (RIE) process using $\mathrm{O}_{2}$ and $\mathrm{SF}_{6}$ (5:1 ratio) plasma was performed to roughen the surface of polyimide substrate and cones. This further increases the surface area and improves the metal adhesion on polyimide. The traces and electrodes were patterned using a lift-off process; a titanium (Ti) adhesion layer followed by gold ( $\mathrm{Au})$ were deposited using evaporation process. A thin sacrificial $\mathrm{Ti}$ layer was also deposited on the gold as a means to visually identify successful etch of the top polyimide layer. (Fig. 1(c)). A 5- $\mu$ m polyimide (PI-2611) was then spin coated and cured in oven. An etch mask was patterned using a 6 - 
(a)

(b)

(c)

(d)

(e)

(f)

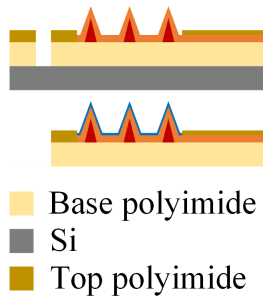

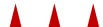

c) $M$ $M \Lambda$
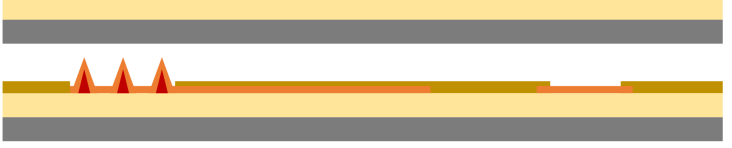

Fabrication process of the flexible 3D microelectrode arrays. (a) Base polyimide coating. (b) Photodefinable polyimide coating and patterning. (c) $\mathrm{Ti} / \mathrm{Au} / \mathrm{Ti}$ evaporation and patterning. (d) Top polyimide coating and etching. (e) Laser dicing. (f) Device release, assembly and electropolymerization of PEDOT:PSS.

$\mu \mathrm{m}$ thick positive photoresist (PR) and the top polyimide was then etched using RIE to expose the electrode and connector sites (Fig. 1(d)). The MEAs were subsequently diced using a femtosecond laser (Fig. 1(e)). A 32-channel Omnetics connector was then connected to each array using anisotropic conductive film (ACF) (Fig. 1(f)).

To further improve the electrical performance, a PEDOT:PSS layer was deposited onto the gold microelectrodes via electropolymerization [6]. An aqueous dispersion of 10mM 3,4-Ethylenedioxythiophene (EDOT) added to $2.0 \mathrm{~g} / 100 \mathrm{~mL}$ sodium polystyrene sulfonate (NaPSS) was used as electrolyte to electropolymerize PEDOT:PSS under galvanostatic conditions in a two-electrode setup, where the gold electrode array served as working electrode and a platinum mesh as counter/reference electrode. A constant current of current density $0.5 \mathrm{~mA} / \mathrm{cm}^{2}$ was applied for $5 \mathrm{~min}$ to deposit a layer of PEDOT:PSS under room temperature.

The PEDOT:PSS coated 3D polyimide micro-cones were successfully fabricated in electrodes with various footprint sizes (from $200 \mu \mathrm{m} \times 200 \mu \mathrm{m}$ to $5 \mu \mathrm{m} \times 5 \mu \mathrm{m}$ ). An optical image of fabricated MEA is shown in Fig. 2(a). The surface morphology of PEDOT:PSS covered cones was examined with a field emission scanning electron microscope (SEM), and an example of $20 \mu \mathrm{m} \times 20 \mu \mathrm{m}$ electrode is shown in Fig. 2(b). The conical shape of the 3D micro-structure is achieved by tuning the exposure and development process for the photodefinable polyimide. The addition of 3D microcones results in an increase in ESA by $150 \%$ to $250 \%$ for different footprint sizes. Electrodeposited PEDOT:PSS uniformly covered the 3D micro-cones, forming a rough and porous coating to further enhance the ESA. The crosssectional view in Fig. 2(c) clearly reveals the different layers of the electrode: the substrate and cone made of polyimide, thin trace metal, and the top PEDOT of the thickness in the order of $1 \mu \mathrm{m}$.
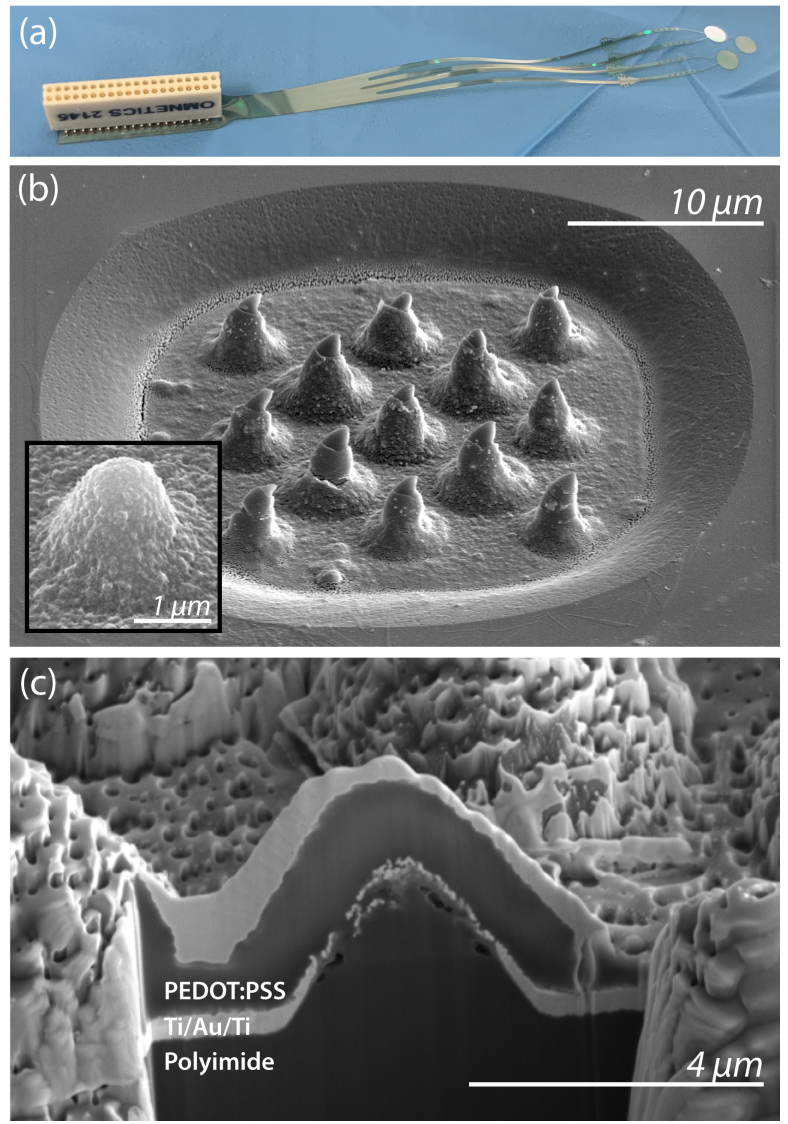

Fig. 2. Optical and electron microscopy. (a) An optical image of the fabricated 3D MEA. (b) A SEM image of a $20 \mu \mathrm{m} \times 20 \mu \mathrm{m}$ electrode pad with 13 cones on top to increase the effective surface area. The inset picture shows the surface contains nano features and porosity after PEDOT:PSS electropolymerization. (c) The SEM of focused ion beam (FIB) cross-section of the electrode site showing multiple layers of the device. A thin layer of tungsten was deposited on top to protect the surface during FIB.

\section{Characterization AND EMG MEASUREMENT}

\section{A. Electrochemical characterization}

In order to evaluate the improvement in electrical performance from the 3D structure and porous PEDOT:PSS coating, impedance at $1 \mathrm{kHz}$ of planar and 3D electrodes both with gold and PEDOT:PSS finishing layers were obtained in a 2-electrode setup in physiological saline solution $(0.9 \%$ $\mathrm{NaCl}$ ) using a $\mathrm{Ag} / \mathrm{AgCl}$ reference electrode. We compared the average impedance $(n=8)$ of electrode sites across eight different sizes as shown in Fig. 3. Electrodes with 3D microcones show a significant impedance reduction across all footprint sizes for both gold and PEDOT:PSS surface finishes. When compared to the gold electrodes, the PEDOT:PSS treatment further reduces the impedance of the 3D microcone electrodes which aligns with previous observation [6]. For $10 \mu \mathrm{m} \times 10 \mu \mathrm{m}$ recording sites, there was a $99.3 \%$ reduction in impedance of the PEDOT:PSS coated 3D micro-cone electrodes as compared to the planar gold electrodes. We are able to achieve high-density recording sites using the $3 \mathrm{D}$ micro-cone structures as the average impedance of the smallest test size for 3D micro-cones $(5 \mu \mathrm{m} \times 5 \mu \mathrm{m})$ was 146.5 $\mathrm{kOhm}$, which was the same as the impedance for a planar gold electrode that was $\sim 250$ times larger $(80 \mu \mathrm{m} \times 80 \mu \mathrm{m})$. 


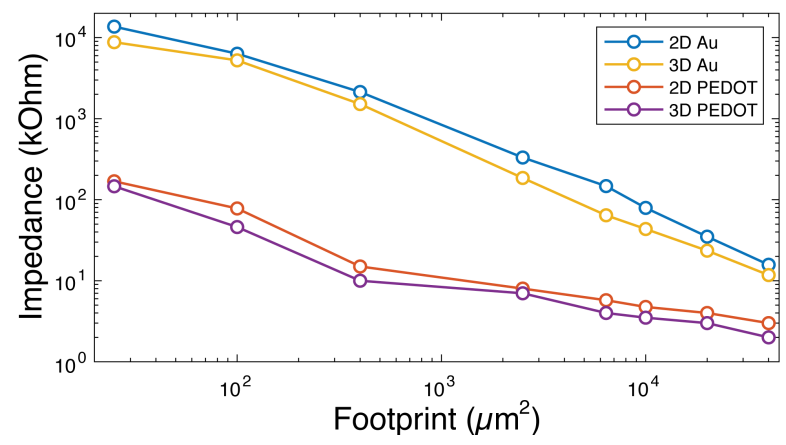

Fig. 3. Average impedance $(\mathrm{n}=8)$ at $1 \mathrm{kHz}$ across eight footprint sizes for planar and 3D electrodes with gold and PEDOT:PSS finishing layers.
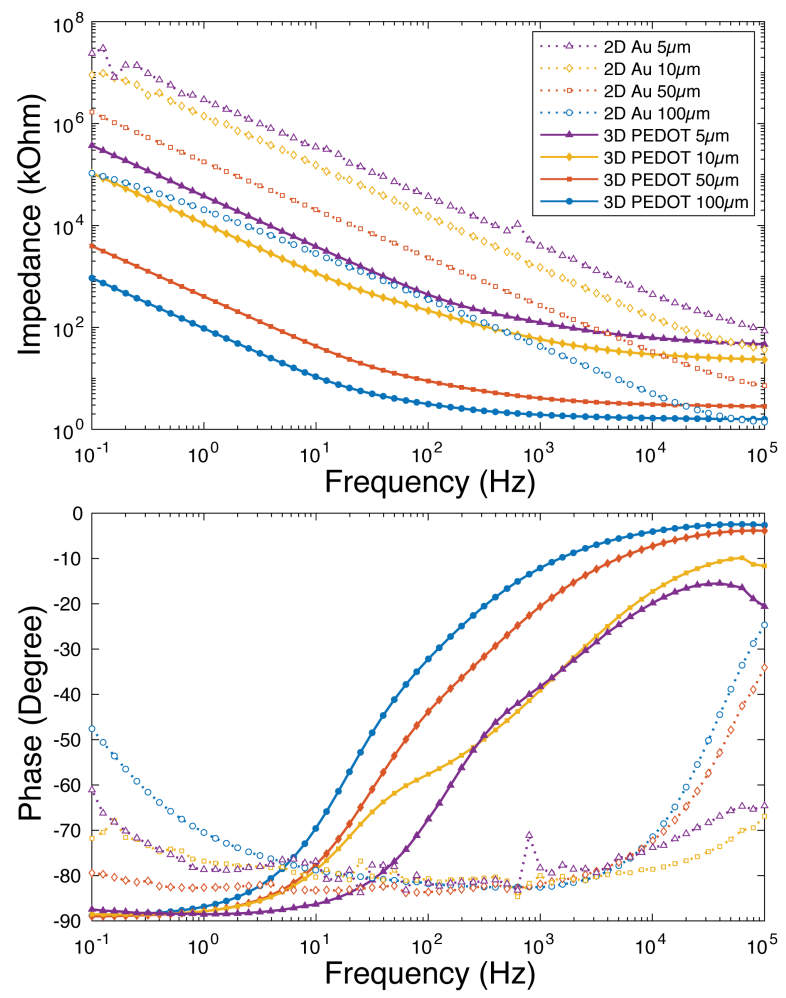

Fig. 4. EIS measurement of 3D PEDOT:PSS coated microelectrodes and 2D gold microelectrodes with four different footprint sizes. Legend applies to both graphs; the number values correspond to the side lengths of the square electrode site.

We further validated the decrease of impedance by comparing the electrochemical impedance spectroscopy (EIS) results between planar gold and 3D PEDOT:PSS coated micro-cone electrodes with representative footprint sizes, as shown in Fig. 4. The experiments were conducted in saline solution with a 3-electrode system with $\mathrm{Ag} / \mathrm{AgCl}$ reference and platinum mesh counter electrodes. The changes in impedance magnitude are consistent with our results at 1 $\mathrm{kHz}$, as the 3D micro-cones together with PEDOT coating significantly reduce the impedance value over the tested frequency range from $0.1 \mathrm{~Hz}$ to $100 \mathrm{kHz}$ for all sizes.

Cyclic voltammetry (CV) measurements were also carried out with the same 3 -electrode setup to determine the charge storage capacity (CSC) of the fabricated 3D micro-cone electrodes. The $\mathrm{CV}$ results of 3D micro-cone electrode with footprint of $200 \mu \mathrm{m} \times 200 \mu \mathrm{m}$ is shown in Fig. 5 and the CSC

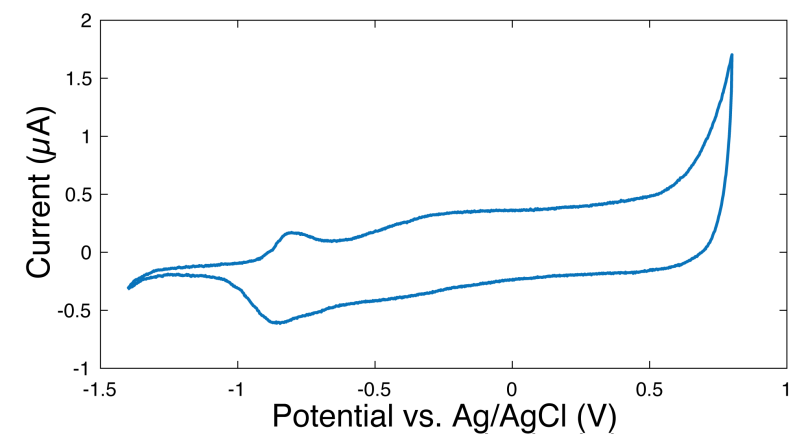

Fig. 5. Cyclic Voltammetry of PEDOT:PSS coated 3D micro-cone electrode with footprint size of $200 \mu \mathrm{m} \times 200 \mu \mathrm{m}$ in saline solution, swept at $100 \mathrm{mV} / \mathrm{s}$.

is calculated to be $34.2 \mathrm{mC} / \mathrm{cm}^{2}$. This demonstrates its feasibility for stimulation application with the CSC comparable or better than some of the published work [14], [15].

\section{B. EMG measurement and data analysis}

The fabricated 3D MEAs with micro-cones were utilized to record EMG activity from digastric muscles of anesthetized mice. All procedures were approved by the Emory University Institutional Animal Care and Use Committee. Anesthesia was induced in 2 adult C57BL/6J male mice using $4 \% \mathrm{vol} / \mathrm{vol}$ isoflurane in oxygen gas and maintained at appropriate levels throughout the procedure and data collection. An incision was made under the jaw, and skin was removed to reveal the digastric muscle. The electrode array was placed on the muscle's surface to collect spontaneouslyoccurring motor unit activity that was amplified via a 32channel digital amplifier headstage (RHD2132; Intan Technologies). EMG signals were recorded on a computer at 30 kHz through a RHD USB Interface Board (Part C3100; Intan Technologies).

Waveforms from different motor units were identified using standard methods that use principal component analysis (PCA) and k-means to cluster waveforms with similar shapes [16]. The amplitude of each spike cluster was measured by the average peak of each waveform in that cluster. The noise level of each channel was measured by the root mean square (RMS) of a one-minute section of signal not containing any spikes. The SNR for each spike was then computed by dividing the amplitude of each spike cluster by the noise level on the corresponding channel.

$$
S N R=\left|\frac{V_{\text {peak }}}{\text { noise }_{R M S}}\right|
$$

High-quality EMG recordings were successfully obtained from mice with the low-impedance 3D MEAs, in which both single and multiple motor units were observed (Fig. 6). In single motor unit recording, the device were able to detect small differences in the action potential's timing and amplitude, which indicate that the electrode has high temporal and spatial resolution to distinguish the different locations of electrode contacts relative to the active muscle fibers. These variations across channels allow multiple motor units to be isolated from single recordings. The multiple motor units recording further showed that our device has significantly higher SNR compared to previously published work [11]. 


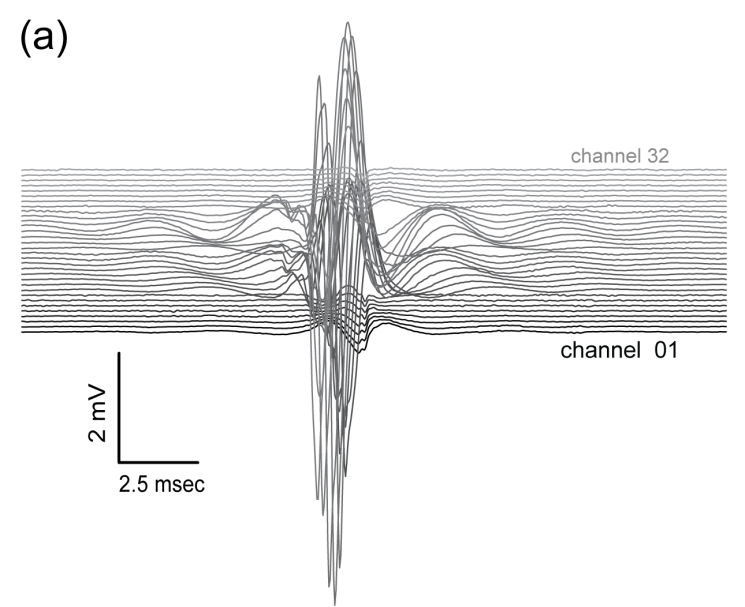

approach for the successful acquisition and analysis of single motor unit activity.

\section{ACKNOWLEDGMENT}

This work was funded by National Institutes of Health Grant R01 NS109237, the McKnight Foundation and the Simons Foundation as part of the Simons-Emory International Consortium on Motor Control, and was performed in part at the Georgia Tech Institute for Electronics and Nanotechnology.

\section{REFERENCES}

[1] V. M. Tysseling, L. Janes, R. Imhoff, K. A. Quinlan, B. Lookabaugh, S. Ramalingam, C. Heckman, and M. C. Tresch, "Design and evaluation of a chronic emg multichannel detection system for long-term recordings of hindlimb muscles in behaving mice," J. Electromyogr. Kinesiol., vol. 23, no. 3, pp. 531-539, 2013.

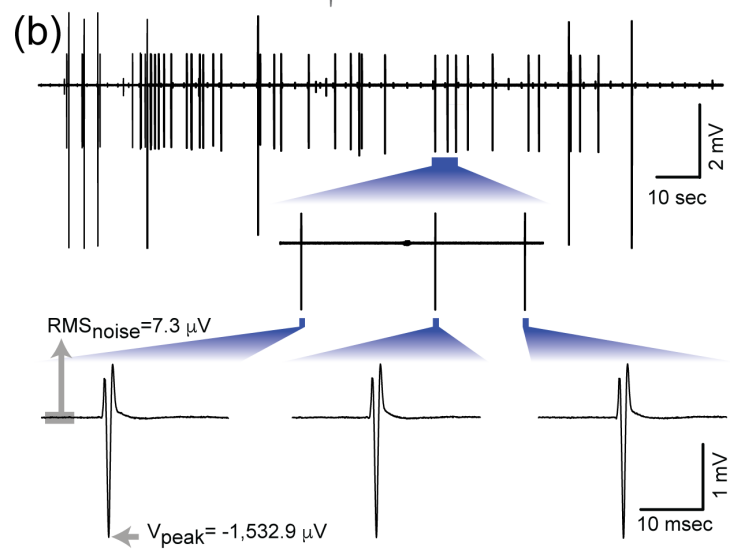

[2] G. L. Warren, C. P. Ingalls, S. J. Shah, and R. Armstrong, "Uncoupling of in vivo torque production from emg in mouse muscles injured by eccentric contractions," J. Physiol., vol. 515, no. 2, pp. 609-619, 1999.

[3] S. J. Sober, S. Sponberg, I. Nemenman, and L. H. Ting, "Millisecond spike timing codes for motor control," Trends Neurosci., vol. 41, no. 10, pp. 644-648, 2018.

[4] K. H. Srivastava, C. M. Holmes, M. Vellema, A. R. Pack, C. P. Elemans, I. Nemenman, and S. J. Sober, "Motor control by precisely timed spike patterns," PNAS, vol. 114, no. 5, pp. 1171-1176, 2017.

[5] E. Seker, Y. Berdichevsky, M. R. Begley, M. L. Reed, K. J. Staley, and M. L. Yarmush, "The fabrication of low-impedance nanoporous gold multiple-electrode arrays for neural electrophysiology studies," Nanotechnology, vol. 21, no. 12, p. 125504, 2010.

[6] R. Gerwig, K. Fuchsberger, B. Schroeppel, G. S. Link, G. Heusel, U. Kraushaar, W. Schuhmann, A. Stett, and M. Stelzle, "Pedotcnt composite microelectrodes for recording and electrostimulation applications: fabrication, morphology, and electrical properties," Front. Neuroeng., vol. 5, p. 8, 2012.

Fig. 6. High-resolution EMG recordings with micro-cone MEAs. (a) Example recording of a single motor unit from a mouse digastric muscle. Here, a single action potential is detected by roughly half of the electrode array's 32 channels. (b) Recording of multiple motor units from a single MEA channel. Top, recording from the digastric muscle during an epoch in which two different motor units distinguished by different amplitudes were recorded simultaneously. Bottom, detail of three individual smaller spikes and their corresponding peak voltage $\left(\mathrm{V}_{\text {peak }}\right)$ and noise level $\left(\mathrm{RMS}_{\text {noise }}\right)$.

We achieved a mean SNR value of $\mathbf{2 0 3 . 3}$ for the motor unit with the smaller spike, as well as a mean SNR of $\mathbf{4 4 7 . 3}$ for the motor unit with the larger spike (Fig. 6(b) top).

\section{CONCLUSION}

In this paper, we present a flexible and bio-compatible microelectrode array with 3D micro-cones and PEDOT:PSS coating that gives a high SNR in in vivo experiments. Through the proposed novel fabrication process, we built micro-cones with photodefinable polyimide that increases the effective surface area up to $250 \%$. Electrochemical characterizations were performed for electrodes with various sizes, where the impedance of our device consistently outperform benchmark devices in tested frequency range. Specifically, the impedance at $1 \mathrm{kHz}$ is reduced by two orders of magnitude. The cyclic voltammetry results show a CSC value of $34.2 \mathrm{mC} / \mathrm{cm}^{2}$, which suggest our device as a good candidate for stimulation applications. The $3 \mathrm{D}$ micro-cone electrodes were successfully implanted in mouse digastric muscles to obtain acute data, where a SNR up to 447.3 was measured through multiple motor units recording. With the high SNR achieved, our device provides a promising

[7] D. A. Koutsouras, A. Hama, J. Pas, P. Gkoupidenis, B. Hivert, C. Faivre-Sarrailh, E. Di Pasquale, R. M. Owens, and G. G. Malliaras, "Pedot:pss microelectrode arrays for hippocampal cell culture electrophysiological recordings," MRS Commun., vol. 7, no. 2, pp. 259-265, 2017.

[8] T. Chung, J. Wang, J. Wang, B. Cao, Y. Li, and S. Pang, "Electrode modifications to lower electrode impedance and improve neural signal recording sensitivity," J. Neural Eng., vol. 12, no. 5, p. 056018, 2015.

[9] S. Beach, S. Grundeen, A. Doyle, and L. Theogarajan, "Fabrication and validation of flexible $3 \mathrm{~d}$ pillar electrodes for neural electrophysiological recording," $E R X$, vol. 2, no. 2, p. 025025, 2020.

[10] Y. Rui, J. Liu, Y. Wang, and C. Yang, "Parylene-based implantable pt-black coated flexible 3-d hemispherical microelectrode arrays for improved neural interfaces," Microsyst. Technol., vol. 17, no. 3, pp. 437-442, 2011.

[11] M. Zia, B. Chung, S. Sober, and M. S. Bakir, "Flexible multielectrode arrays with 2-d and 3-d contacts for invivo electromyography recording," IEEE Trans. Compon. Packag. Manuf. Technol., vol. 10, no. 2, pp. 197-202, 2020.

[12] C. Nick, S. Quednau, R. Sarwar, H. Schlaak, and C. Thielemann, "High aspect ratio gold nanopillars on microelectrodes for neural interfaces," Microsyst. Technol., vol. 20, no. 10-11, pp. 1849-1857, 2014.

[13] J. T. Robinson, M. Jorgolli, A. K. Shalek, M.-H. Yoon, R. S. Gertner, and H. Park, "Vertical nanowire electrode arrays as a scalable platform for intracellular interfacing to neuronal circuits," Nat. Nanotechnol., vol. 7, no. 3, pp. 180-184, 2012.

[14] S. Shin, J. Kim, J. Jeong, T. M. Gwon, G. J. Choi, S. E. Lee, J. Kim, S. B. Jun, J. W. Chang, and S. J. Kim, "High charge storage capacity electrodeposited iridium oxide film on liquid crystal polymer-based neural electrodes," Sens. Mater, vol. 28, no. 3, pp. 243-260, 2016.

[15] W. Yang, Y. Gong, C.-Y. Yao, M. Shrestha, Y. Jia, Z. Qiu, Q. H. Fan, A. Weber, and W. Li, "A fully transparent, flexible pedot: Pss-ito-agito based microelectrode array for ecog recording," Lab Chip, vol. 21, no. 6, pp. 1096-1108, 2021.

[16] S. J. Sober, M. J. Wohlgemuth, and M. S. Brainard, "Central contributions to acoustic variation in birdsong," J. Neurosci., vol. 28, no. 41, pp. 10370-10379, 2008. 